\title{
Eletrocardiografia computadorizada em cavalos Puro Sangue Lusitano submetidos a exercício físico
}

[Computerized electrocardiography in Lusitano horses submitted to exercise]

\author{
${\text { A. } \text { Melchert }^{1} \text {, C.B. Laposy }}^{1}$, V.H.B. Guasi ${ }^{2}$, H.F.D. Valle $^{2}$, G.C. Santos ${ }^{2}$ \\ ${ }^{1}$ Faculdade de Ciências Agrárias - Universidade do Oeste Paulista - Unoeste, Presidente Prudente, SP \\ ${ }^{2}$ Aluno de graduação - Universidade do Oeste Paulista - Unoeste - Presidente Prudente, SP
}

\begin{abstract}
RESUMO
Foram estudados 24 cavalos atletas hígidos, machos, adultos, da raça Puro Sangue Lusitano, submetidos a esforço físico de trote e galope de rotina do animal, com o objetivo de avaliar a configuração do traçado do eletrocardiograma (ECG) em repouso, mediante eletrocardiografia computadorizada, bem como os efeitos do exercício físico sobre os parâmetros clínicos e eletrocardiográficos destes animais. Foram avaliados exame físico e ritmo cardíaco antes e imediatamente após o exercício físico, assim como as distintas configurações de ondas p e complexos QRS, obtidas ao ECG de repouso. Após o treinamento, ocorreram taquicardia e taquipneia. O ritmo predominante no repouso foi o sinusal e, após o exercício, a taquicardia sinusal. Na morfologia das ondas, encontraram-se quatro configurações distintas para a onda $p$, e seis para o complexo QRS. Dentre as arritmias cardíacas em repouso, houve uma ocorrência de bloqueio atrioventricular de segundo grau, que desapareceu após exercício. Conclui-se que o traçado do ECG computadorizado de cavalos atletas Puro Sangue Lusitano revela distintas morfologias de ondas p e complexos QRS e baixa ocorrência de arritmias.
\end{abstract}

Palavras-chave: equinos, eletrocardiografia computadorizada, avaliação clínica, exercício físico

\begin{abstract}
In order to assess the configuration of the electrocardiogram (ECG) at rest obtained through computerized electrocardiography and the effects of exercise on clinical and electrocardiographic parameters, 24 healthy male Pure-bred adult Lusitano horses submitted to daily trot and gallop training were studied. Physical evaluation and cardiac rhythm were evaluated before and immediately after exercise, as well the different $p$ waves and QRS complexes, obtained from rest ECG. Tachycardia and tachypnea were observed after training. The predominant rest rhythm was sinus rhythm and, after exercise, sinus tachycardia. In the wave morphology, four distinct configurations were found for the $p$ wave, and six different settings for the QRS complex. Second degree atrioventricular block was observed once during rest, and it disappeared after exercise. In conclusion, computerized ECG in Pure-bred Lusitano horses reveals distinct morphologies of p waves and QRS complexes, and cardiac arrhythmias were rare.
\end{abstract}

Keywords: equine, computerized electrocardiography, clinical evaluation, physical exercise

\section{INTRODUÇÃO}

O emprego de testes para a avaliação do desempenho atlético, juntamente com as respostas fisiológicas obtidas pela ação do exercício e do treinamento, pode ser uma valiosa ferramenta para maximização dos resultados obtidos nas competições. O programa de treinamento deixa de ser realizado somente de

Recebido em 2 de março de 2011

Aceito em 27 de janeiro de 2012

E-mail: alessandravet@unoeste.br maneira empírica tornando-se um processo técnico, com embasamento clínico e fisiológico (Lindner et al., 2006; Erck et al., 2007). Além disso, os testes a campo possuem utilidade clínica no monitoramento de enfermidades cardíacas, respiratórias e músculo-esqueléticas (Couroucé, 1999).

A participação de atletas em eventos cada vez mais exaustivos fez com que o conhecimento da 
resposta cardíaca frente a estes estímulos seja essencial para a segurança deles (Dumont et al., 2011). Na medicina equina, há a preocupação com a queda de desempenho em decorrência das disfunções cardiovasculares, responsáveis por alterações que se estendem desde uma intolerância ao exercício até a morte súbita (Kiryu et al., 1999).

As anormalidades cardíacas são referidas como a terceira maior causa de queda de desempenho em cavalos atletas, atrás apenas das alterações musculoesqueléticas e respiratórias, sendo importante o registro do eletrocardiograma (ECG) (Diniz et al., 2011). O ECG é um método pouco oneroso, não invasivo e de fácil realização a campo, além de ser o método mais seguro para diagnóstico das arritmias cardíacas. Deve ser acompanhado de um exame clínico acurado do sistema cardiovascular (Fernandes et al., 2004).

Em equinos, o ECG é válido para a determinação da frequência, do ritmo e dos tempos de condução. A avaliação dos complexos para a determinação do tamanho do coração é limitada, visto que a gênese do ECG é diferente da que ocorre no homem e em pequenos animais. Por isso, a determinação de arritmias e distúrbios de condução ainda é a principal indicação de ECG na espécie (Fernandes et al., 2004). Outra contribuição importante, sugerida por Babusci e López (2006), é a possibilidade de detecção de distúrbios eletrolíticos.

Muitos pesquisadores relataram variações no ritmo cardíaco de equinos, indicando que determinadas espécies são muito mais susceptíveis a arritmias cardíacas. Portanto, para correta avaliação e interpretação do ECG, é necessário conhecer os padrões eletrocardiográficos relacionados com a espécie, a raça, e até mesmo com a idade dos animais (Fernandes et al., 2004). Os parâmetros eletrocardiográficos apresentaram modificações entre as diferentes raças equinas, sendo necessário que cada raça tenha seu padrão específico (Illera et al. 1987; Ayala et al., 1995; Fernandes et al., 2004; Diniz et al., 2008).

Montado há cerca de 5000 anos, o cavalo Lusitano é o mais antigo cavalo de sela do mundo. É um animal versátil, cuja docilidade, agilidade e coragem lhe permitem hoje competir em quase todas as modalidades do moderno esporte equestre, participando de jogos panamericanos e equipes olímpicas, representando vários países do mundo (Cordeiro, 2005). Entretanto, não foram encontrados na literatura trabalhos que avaliem o ECG e os efeitos do treinamento físico nesta espécie.

Sendo assim, o presente estudo objetivou avaliar o ritmo cardíaco e a configuração do traçado eletrocardiográfico de cavalos atletas da raça Puro Sangue Lusitano em repouso, obtidos mediante eletrocardiografia computadorizada, bem como os efeitos do exercício físico sobre os parâmetros clínicos e eletrocardiográficos destes animais, após treinamento de rotina.

\section{MATERIAL E MÉTODOS}

Após devida aprovação ética pela Instituição de origem, sob o protocolo 294/10, o estudo utilizou 24 animais, da espécie equina, da raça Puro Sangue Lusitano, machos, com idades entre quatro e 12 anos $(5,5 \pm 2,1)$, submetidos a uma rotina diária de exercícios físicos de trote e galope. O tempo de treinamento dos animais oscilou entre 15 e 25 minutos $(20,04 \pm 2,26)$.

Foram avaliados: coloração da mucosa oral; tempo de preenchimento capilar (TPC), por compressão digital da mucosa oral e posterior inspeção; frequência respiratória $(f)$, por inspeção dos movimentos da caixa torácica; auscultação pulmonar e cardíaca; eletrocardiograma (ECG) para determinar o ritmo e a frequência cardíaca (FC). As avaliações do exame físico e do ECG foram realizadas antes e imediatamente após o exercício físico.

$\mathrm{Na}$ avaliação da FC, foi considerado o intervalo entre 25 a 46 batimentos por minuto (bpm) para normalidade. Este critério baseou-se nos valores de referência de 25 a 40 batimentos por minuto (bpm), descritos por Feitosa (2004), além de 6 bpm acima da frequência cardíaca de repouso, que caracteriza algum tipo de estresse (Craig e Nunan, 1998), como, por exemplo, o estresse de manipulação do animal para registro do ECG. Para a FR, foram considerados os valores estabelecidos por Feitosa (2004), que variam entre 10 a 14 movimentos por minuto (mpm) em cavalos adultos. 
O ECG foi realizado em aparelho eletrocardiógrafo computadorizado (ECG PC $\left.\mathrm{TEB}^{\circledR}\right)$. Os animais foram posicionados em estação, em cima de piso emborrachado para isolamento elétrico, com os membros anteriores e posteriores paralelos entre si, com eletrodos fixados na região do olécrano nos membros anteriores, e na região da patela nos membros posteriores, umedecidos com álcool para melhor contato com a pele (Neto, 2004).

Foram registradas as derivações bipolares (I, II e III) e unipolares aumentadas (aVR, aVL e aVF), em 25 e $50 \mathrm{~mm} / \mathrm{seg}$, calibração da milivoltagem em $1 \mathrm{~cm}=1 \mathrm{mV}$. Os parâmetros avaliados no ECG foram a FC e o ritmo cardíaco, determinados antes e após o exercício, bem como a análise morfológica das ondas $\mathrm{P}$ e dos complexos QRS, avaliados antes do exercício.

Para avaliação estatística dos dados obtidos antes e depois do exercício, foi utilizado o teste de T pareado, considerando-se um nível de significância de 5\% (Pagano e Gavreau, 2004).

\section{RESULTADOS}

A avaliação do exame físico dos animais antes do exercício físico revelou normalidade dos parâmetros TPC e das membranas mucosas (Feitosa, 2004). Estes parâmetros não apresentaram alterações após o exame físico. A ausculta cardiorrespiratória foi normal em todos os cavalos, não se observando a presença de estertores, sopro ou arritmias em nenhum animal.

A frequência respiratória $(f)$ oscilou entre 12 e 16 mpm $(14,5 \pm 1,98)$ antes do exercício físico. Após o exercício, a FR oscilou entre 20 e $42 \mathrm{mpm}$
$(46,5 \pm 13,8)$, sendo observado um aumento na FR de extrema significância $(p<0,0001)$. Foi observada a presença de taquipneia, após o exercicio, em todos os animais estudados após o exercício, segundo os valores descritos por Feitosa (2004).

$\mathrm{Na}$ avaliação da frequência cardíaca (FC) antes do exercício, os valores oscilaram entre 27 e $49 \mathrm{bpm}(36,2 \pm 6,3)$, sendo que 23 cavalos apresentaram FC normal, enquanto apenas em um foi constatada taquicardia, com FC de 49 bpm, acima dos $46 \mathrm{bpm}$ considerados neste estudo. Após o exercício, foi observado um aumento na FC de extrema significância $(\mathrm{P}<0,0001)$, sendo que 20 cavalos apresentaram taquicardia e quatro apresentaram FC normal. A FC variou entre 35 e 80 bpm $(57,7 \pm 11,6)$.

$\mathrm{Na}$ avaliação do ritmo cardíaco, observou-se a ocorrência de ritmo sinusal em 21 animais $(87,5 \%)$, arritmia sinusal em um animal $(4,16 \%)$, bloqueio átrio-ventricular de $2^{\circ}$ grau (BAV-2) intermitente (Fig. 1) em um animal $(4,16 \%)$ e taquicardia sinusal em um cavalo $(4,16 \%)$, antes do exercício. Após o exercício, o ritmo observado foi taquicardia sinusal em 20 cavalos $(83,3 \%)$ e ritmo sinusal em quatro animais $(16,7 \%)$.

Em relação à morfologia das ondas, a onda $p$ apresentou quatro formas distintas (Fig. 2), representadas na Tab. 1 .

Em relação à morfologia do complexo QRS, foram obtidas seis formas distintas (Fig. 3), representadas na Tab. 2.

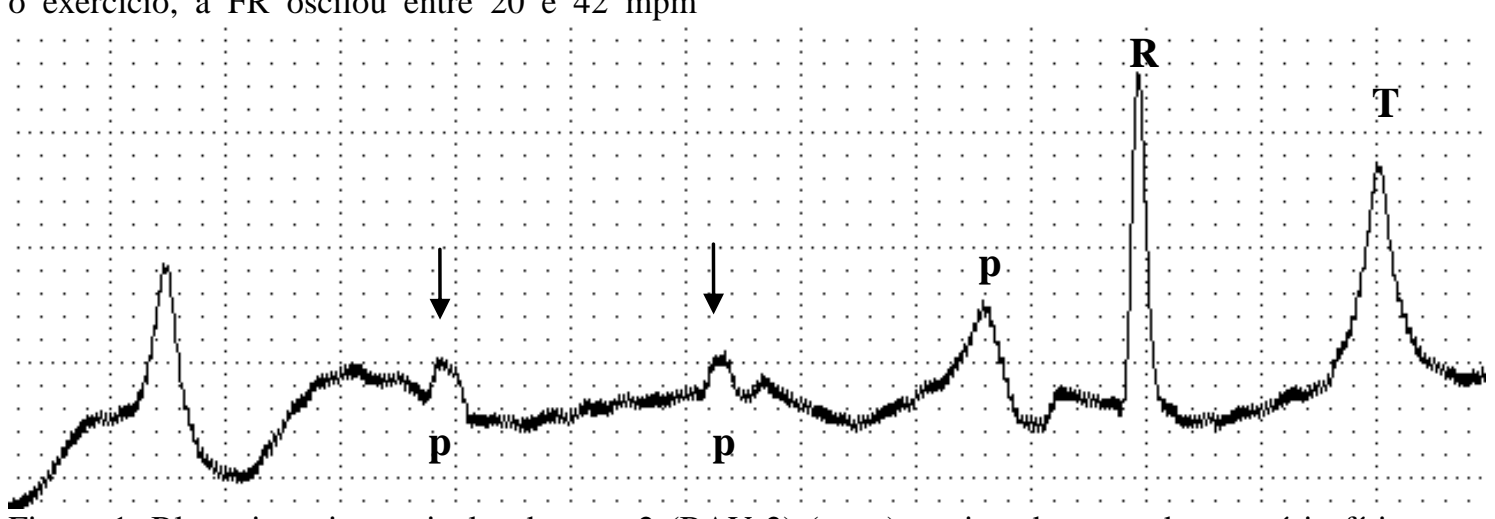

Figura 1. Bloqueio atrioventricular de grau 2 (BAV 2) (setas), registrado antes do exercício físico, em cavalos atletas da raça Puro Sangue Lusitano. 
Tablela 1. Morfologias distintas de ondas p, observadas em cavalos Puro Sangue Lusitanos, antes do exercício físico

\begin{tabular}{ll}
\multicolumn{1}{c}{ Tipos de onda P } & \multicolumn{1}{c}{$\mathrm{N}^{\mathrm{o}}$ de animais (\%) } \\
\hline (+ -) P bifásica,P1(positiva) e P2(negativa) & 3 animais $(12,5 \%)$ \\
(- +) P bifásica,P1(negativa) e P2(positiva) & 3 animais $(12,5 \%)$ \\
(+) P única (positiva) & 4 animais $(16,7 \%)$ \\
$(++)$ p bífida (positiva) & 14 animais $(58,3 \%)$ \\
\hline
\end{tabular}

P1- $1^{a}$ porção da onda p; P2- $2^{a}$ porção da onda p.

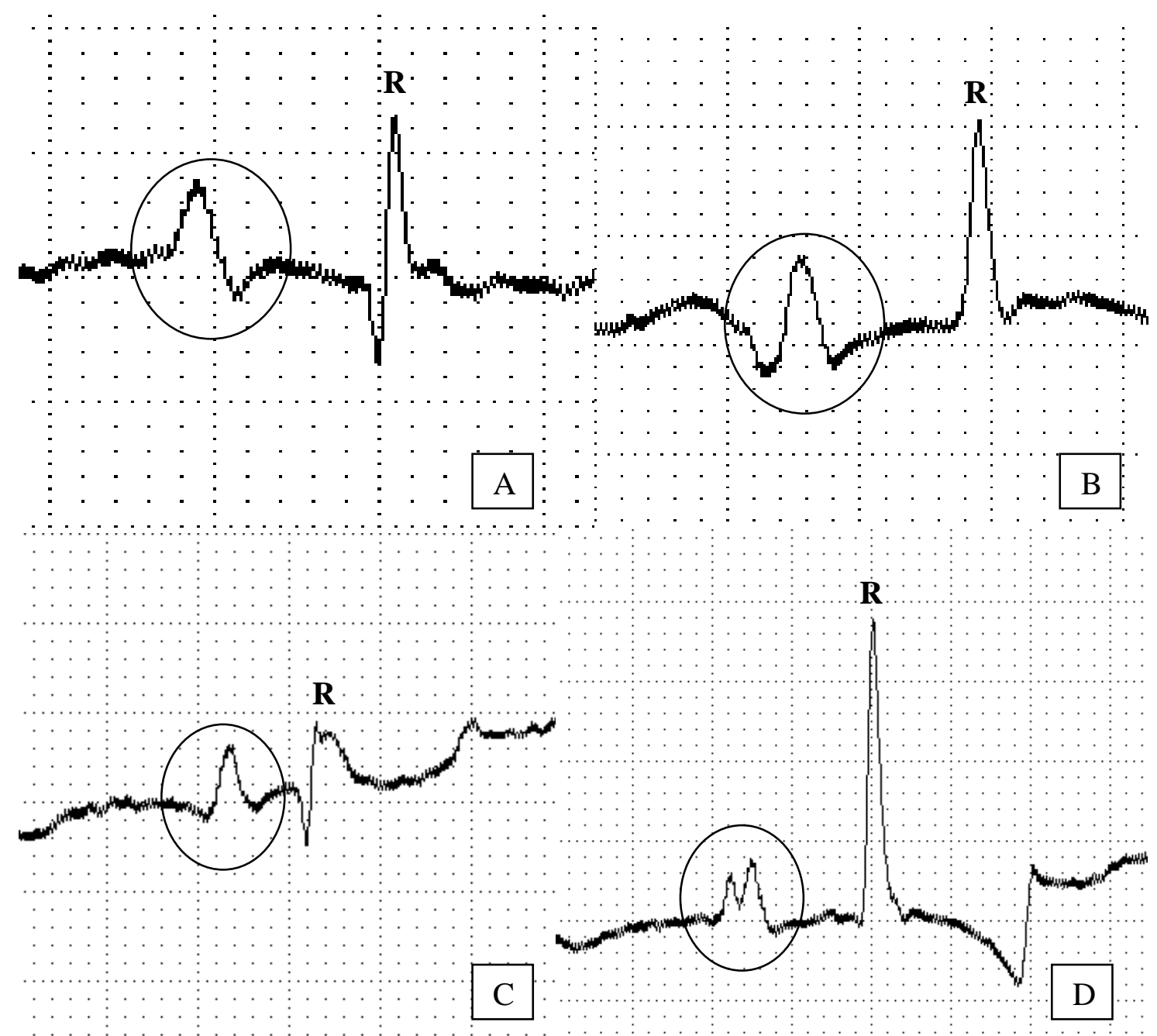

Figura 2. Diferentes morfologias de onda $\mathrm{p}$ (circundadas) registradas em cavalos atletas da raça Puro Sangue Lusitano: A) onda p bifásica com $1^{\mathrm{a}}$ porção $(\mathrm{P} 1)$ positiva e $2^{\mathrm{a}}$ porção $(\mathrm{P} 2)$ negativa; $\left.\mathrm{B}\right)$ onda $\mathrm{p}$ bifásica com P1 negativa e P2 positiva; C) única e positiva; D) bífida positiva (++). 

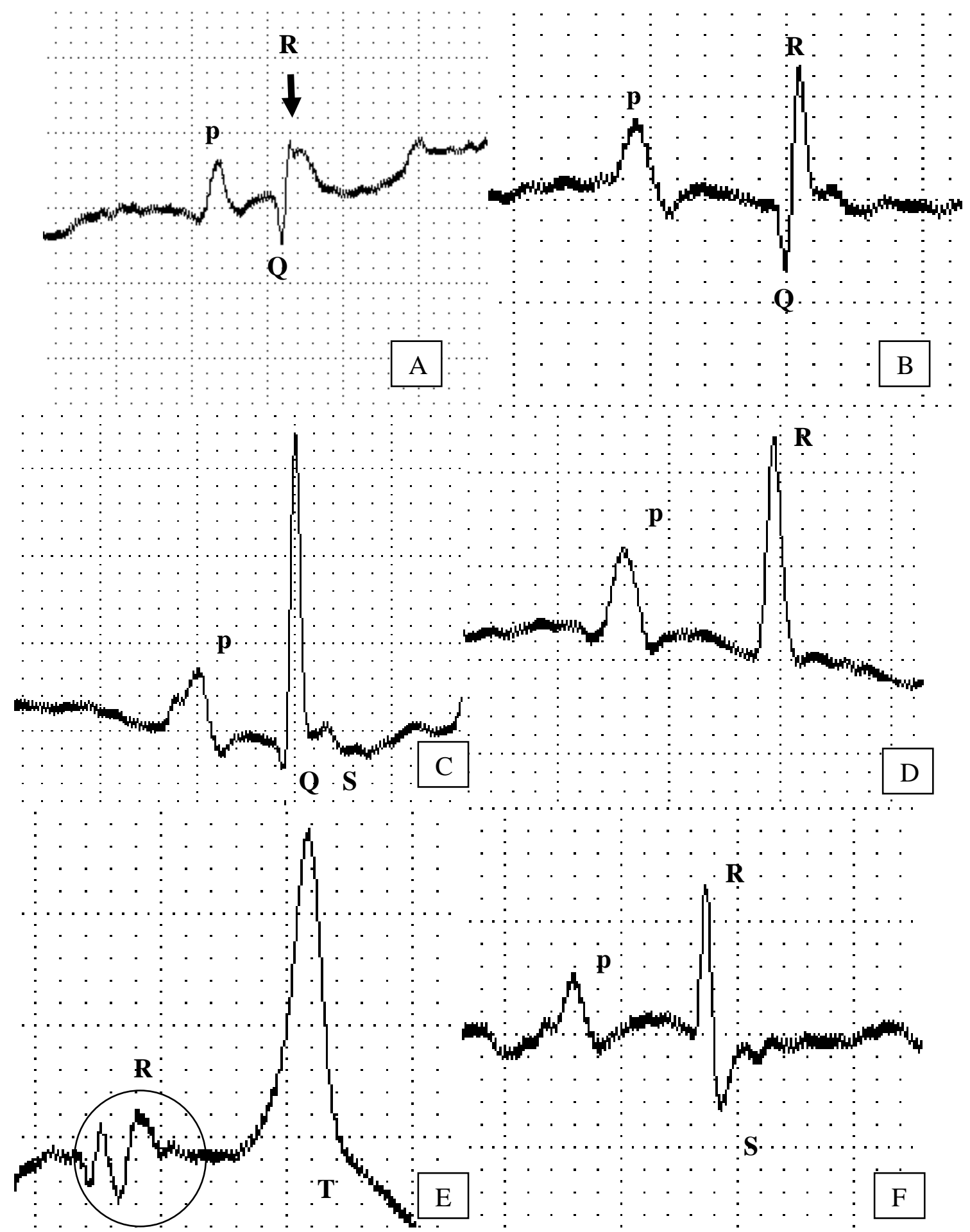

Figura 3. Diferentes morfologias de complexos QRS registradas em cavalos atletas da raça Puro Sangue Lusitano: A) tipo QR (ausência de onda S), com onda R fenestrada (R fenestrado) (seta); B) tipo QR (ausência de onda S); C) tipo QRS (presença de QRS); D) Tipo R (ausência de ondas Q e S) ; E) tipo R (ausência de ondas Q e S ) com R fenestrado (circundado); F) tipo RS (ausência de onda Q). 
Tabela 2. Morfologias distintas de complexos QRS, observadas em cavalos Puro Sangue Lusitano, antes do exercício físico

\begin{tabular}{ll}
\multicolumn{1}{c}{ Tipos de Complexo QRS } & \multicolumn{1}{c}{$\mathrm{N}^{\circ}$ de animais } \\
\hline (QRS): presença de QRS & 3 animais $(12,5 \%)$ \\
(QR): ausência de onda S & 12 animais $(50 \%)$ \\
(RS): ausência de onda Q & 1 animal $(4,2 \%)$ \\
(R): ausência de ondas Q e S & 5 animais $(20,8 \%)$ \\
(Rf): ausência de ondas Q e S e onda R fenestrada & 1 animal $(4,2 \%)$ \\
(QRf): ausência de onda S e onda R fenestrada & 2 animais $(8,3 \%)$ \\
\hline
\end{tabular}

\section{DISCUSSÃO}

Dentre as alterações observadas no exame dos animais após o exercício físico, destacou-se a ocorrência de aumento das frequências respiratória $(f)$ e cardíaca (FC), em $100 \%$ e $83,3 \%$ dos cavalos avaliados, respectivamente. A presença de taquipneia e taquicardia após o treinamento é justificada pela estimulação simpática causada pelo exercício físico. Mediante a estimulação simpática, ocorre o aumento na força de contração do miocárdio, aumentando o fornecimento de sangue para os músculos em contração durante o exercício (Kraemer e Ratamess, 2005).

A média da FC de repouso (antes do exercício) foi de 36,2 bpm. É importante destacar que, em cavalos atletas, de acordo com a raça do animal, diferentes valores de $\mathrm{FC}$ em repouso foram citados na literatura. Na avaliação da FC de equinos de enduro em repouso, foram descritos valores distintos para as raças Puro Sangue Inglês (PSI), Árabe (média de FC de 35,3 e 38,8 bpm, respectivamente), Mangalarga e Mestiça (49 e 51 bpm de média, respectivamente) (Dumont et al., 2010). Estes resultados ressaltam a importância do conhecimento dos parâmetros eletrocardiográficos de diferentes raças de cavalos, entre estes, o Puro Sangue Lusitano.

$\mathrm{Na}$ avaliação do ritmo cardíaco antes do exercício, observou-se a predominância do ritmo sinusal $(87,5 \%)$, além de arritmia sinusal, BAV-2 intermitente (Fig. 1) e taquicardia sinusal, com uma ocorrência de cada ritmo $(4,16 \%)$. De modo similar, em equinos PSI sobreanos, o ritmo sinusal foi predominante $(94 \%)$, além da ocorrência de arritmia sinusal $(2 \%)$ e taquicardia sinusal (4\%) (Fernandes et al., 2004).

A ausência de arritmias após o treinamento e a ocorrência de apenas um caso de arritmia cardíaca antes do exercício, representada pelo único registro de $\mathrm{BAV}-2$, contrastaram com os dados da literatura, uma vez que diversos pesquisadores relataram variações no ritmo cardíaco de equinos (Mcguirk e Muir, 1985; Vicenzi et al., 2000).

O BAV-2 pode ser uma forma de estímulo vagal (Cárdenas et al., 2009). Raekallio (1992) ao avaliar nove cavalos hígidos por holter, descreveu a ocorrência de períodos de BAV-2 em três animais, causados, aparentemente, por variações do sistema nervoso autônomo (SNA). Deve-se destacar que, neste estudo, o BAV-2 desapareceu após o exercício físico. A frequência cardíaca responde dinamicamente a alterações fisiológicas mediadas pelo SNA por meio de impulsos nervosos eferentes vagais e simpáticos (Kleiger et al., 2005).

Segundo Oakley e Sheffield (2001), o eletrocardiograma do atleta humano foi geralmente normal, entretanto algumas alterações surgiram, como, por exemplo, a bradicardia sinusal. Muitos desses padrões foram normalizados no eletrocardiograma de esforço, o que pôde diferenciar o coração do atleta de uma cardiopatia. Muitas das alterações ao ECG em equinos foram detectadas independentemente de doença ou alteração funcional do coração, sendo atribuídas à alta variação do tônus vagal em condições de repouso, e consideradas benignas ou fisiológicas (Mcguirk e Muir, 1985; Vicenzi et al., 2000).

Foram observadas distintas formas de onda $\mathrm{p}$ neste estudo. A onda $\mathrm{p}$ em equinos apresentou morfologia geralmente variável, o que dificulta sua padronização, pois pode sofrer influência da raça, sexo e idade (Illera et al., 1987). Ayala et al. (1994) descreveram em equinos da raça Andaluz a ocorrência de diferentes morfologias de $\mathrm{p}$, entre elas a $\mathrm{p}$ bífida, $\mathrm{p}$ única e $\mathrm{p}$ bifásica, de modo similar ao encontrado neste estudo. Diniz (2006) descreveu a ocorrência de onda p bífida e 
positiva como a mais frequente, com ocorrência em $53 \%$ dos equinos de salto avaliados. De modo similar neste estudo, esta morfologia de $\mathrm{p}$ foi também a mais frequente, ocorrendo em $58,3 \%$ dos animais avaliados.

No presente trabalho, foram encontradas seis formas distintas de QRS. A morfologia mais frequente na derivação bipolar II foi a do tipo QR (50\%), resultado similar ao relatado por Diniz (2006), em equinos de salto. Estes resultados diferiram dos relatados por Ayala et al. (1994) em estudo em equinos da raça Andaluz, que verificaram a morfologia do tipo QRS como a mais frequente $(58,3 \%)$.

\section{CONCLUSÃO}

Conclui-se que o traçado do ECG computadorizado de cavalos atletas Puro Sangue Lusitano revela distintas morfologias de ondas $\mathrm{p}$ e complexos QRS e baixa ocorrência de arritmias, sendo o ritmo sinusal e a taquicardia sinusal os ritmos cardíacos predominantes em repouso e após o exercício, respectivamente.

\section{REFERÊNCIAS}

AYALA, I.; MONTES, A.; BERNAL, L.J. et al. Electrocardiographic values in Spanish-bred horses of different ages. Aust. Vet. J., v.72, p.225-226, 1995.

AYALA, I.; MONTES, A.M.; FERNADEZ del PALACIO, M.J; et al. Apotaciones al estúdio electrocardiográfico del caballo.An. Vet., v.9, n.10, p.25-35, 1994.

BABUSCI, M.; LÓPEZ, E.F. Sistema cardiovascular. In: BOFFI, F.M. Fisiologia del ejercicio en equinos. Buenos Aires: Inter-Médica, 2006. Cap.4, p.61-85.

CARDENAS, J.J.; LUNA, S.P.L.; TEIXEIRA NETO, F. et al. Comparação entre a lidocaína e a acupuntura no tratamento da taquicardia ventricular induzida com dopamina em equinos anestesiados com halotano. Arq. Bras. Med. Vet. Zootec., v.61, p.777-784, 2009.

CORDEIRO, A.R. Cavalo Lusitano: O filho do vento. Lisboa: Inapa, 2005. 229 p.

COUROUCÉ, A. Field exercise testing for assessing fitness in French Standardbred Trotters. Vet. J., v.157, p.112-122, 1999.

CRAIG N.; NUNAN, M. Entrenamiento del ritmo cardiaco para caballos. Australia Sur: Performance Matters Pty Ltda, 1998.
DINIZ, M.P. Perfil eletrocardiográfico em equinos de salto criados em São Paulo. São Paulo, 2006. Dissertaçăo (mestrado) - Universidade de São Paulo, Departamento de Clínica Médica, Programa de PósGraduação em Clínica Veterinária.

DINIZ, M.P.; MUZZI, R.A.L.; MUZZI, L.A.L. et al. Estudo eletrocardiográfico de equinos da raça Mangalarga Marchador. Arq. Bras. Med. Vet. Zootec. v.60, p.536-542, 2008.

DINIZ, M.P.; MICHIMA, L.E.S.; FERNANDES, W.R. Estudo eletrocardiográfico de equinos de salto sadios. Pesq. Vet. Bras., v.31, p.355-361, 2011.

DUMONT, C.S.B.; MORAES, J.M.; LEITE, C.R. et al. Parâmetros eletrocardiográficos de equinos desclassificados por exaustão em competições de enduro. Arq. Bras. Med. Vet. Zootec., v.63, p.20-27, 2011.

DUMONT, C.S.B.; LEITE, C.R.; MORAES, J.M. et al. Parâmetros eletrocardiográficos de equinos Puro Sangue Árabe submetidos a exercício prolongado de enduro. Cienc. Rural, v.40, p.1966-1973, 2010.

ERCK, E.; VOTION, D.M.; SERTEYN, D. et al. Evaluation of oxygen consumption during field exercise tests in Standardbred trotters. Equine Comp. Exerc. Physiol., v.4, p.43-49, 2007.

FEITOSA, F.L.F. Semiologia veterinária - A arte de diagnóstico. São Paulo: Editora Roca Ltda, 2004, $735 \mathrm{p}$.

FERNANDES, W.R.; LARSSON, M.H.M.A.; ALVES, A..L.G. et al. Características eletrocardiográficas em equinos clinicamente normais da raça Puro Sangue Inglês. Arq. Bras. Med. Vet. Zootec., v.56, p.143-149, 2004.

ILLERA, J.C.; ILLERA, M.; HAMLIN, R.L. Unipolar thoracic electrocardiography that induces QRS complexes of relative uniformity from male horses. Am. J. Vet. Res., v.48, p.1700-1702, 1987.

KIRYU, K.; MACHIDA, N.; KASHIDA, Y. et al. Pathologic and electrocardiographic findings in sudden cardiac death in race horses. J. Vet. Med. Sci., v.61, p.921-928, 1999.

KLEIGER, R.E.; STEIN, P.K.; BIGGER, J.T. Heart rate variability: measurement and clinical utility. Ann. Noninvasive Electrocardiol., v.10, p.88-101, 2005.

KRAEMER, W.J., RATAMESS, N.A. Hormonal responses and adaptations to resistance exercise and training. Sports Med., v.35, p.339-361, 2005.

LINDNER, A.; SIGNORINI, R.; BRERO, L. et al. Effect of conditioning horses with short intervals at high speed on biochemical variables in blood. Equine Vet. J., v.36, Suppl., p.88-92, 2006. 
McGUIRK, S.M.; MUIR, W.W. Diagnosis and treatment of cardiac arrhythmias. Vet. Clin. North Am.: Equine Pract., v.1, p.353-370, 1985.

NETO, D.A. Semiologia do Sistema circulatório de equinos e ruminantes. In: FEITOSA, F.L.F. Semiologia veterinária - A arte de diagnóstico. São Paulo: Editora Roca Ltda, 2004. p.233- 281.

OAKLEY, D.; SHEFFIELD, U.K. The athlete's heart. Heart, v.86, p.72-726, 2001.
PAGANO, M.; GAUVREAU, K. Princípios de Bioestatística. 2.ed. São Paulo: Pioneira Thomson Learning, 2004, 506p.

RAEKALLIO, M. Long term ECG recording with holter monitoring in clinically healthy horses. Acta Vet. Scand., v.33, p.71-75, 1992.

VICENZI, R.C.; LARSSON, M.H.M.A.; FERNANDES, W.R. Parâmetros eletrocardiográficos de equinos clinicamente normais da raça Mangalarga. Parte I: frequência e ritmo cardíaco. Rev. Bras. Med. Vet., v.22, p.71-73, 2000. 